\section{Building survivor activism: An organisational view}

\author{
Shameem Sadiq-Tang, *
}

\section{Introduction}

The purpose of this paper is to share how different models of survivor activism can be built in a clinical charity with a human rights ethos and to set out the value that has come from growing survivor activism organically, based on experiences at Freedom from Torture. The work was formally established as survivor activism eight years ago although it began years before. Since then it continues to evolve and, as a result, there is a strong programme based on collaborative approaches and good practice for safe and informed engagement. From an organisational perspective, it is possible to highlight risk reduction, empowered spaces and enabling survivors of torture to be their own agents of change as key factors in a successful survivor activism programme.

It is widely acknowledged that survivor involvement is important, not only in shaping the rehabilitation and other services they receive but also in advocating for positive change in the wider world. Juan Mendez, former UN Special Rapporteur on Torture, ${ }^{1}$ amongst others, has indicated

1 https://www.freedomfromtorture.org/survivor_ activism

^) Survivor Activism Manager, Freedom from Torture, UK. Shameem has led the Survivor Activism team for eight years.

Correspondence to:

ssadiqtang@freedomfromtorture.org

(Also to find out more about survivor activism). that governments need to be consulting survivors, who are the experts, rather than the top-down method. But, survivor activism can mean many things, and there are questions over how it can best be carried out in an environment already plagued by a lack of resources. At Freedom from Torture, we distinguish survivor activism from the work we also do to engage service users in our own organisation. We see survivor activism as amplifying the voices of survivors of torture in discussions and debates in spaces of influence and decision- making fora. It must go beyond tokenism or consultation. To me, this means that survivors voices, words and narratives are aimed at shaping and changing policy and practice on torture and its impact. It means formally recognising survivor activism as a body of work and allocating resources to support survivors to meaningfully engage in activism. Our survivor activism has not developed in response to research findings based on samples or methodologies, but a genuine belief amongst service users and staff in the value of activism by those with lived experience of torture. It is based on years of working in collaboration with survivors of torture and getting their positive feedback, and simply getting out there and doing and learning along the way.

In a climate of limited funding, organisations have to make difficult decisions about where to invest resources. Freedom from Torture is one of the larger torture rehabilitation centres but we still face these dilemmas. However, a commitment to putting survivors of torture at the heart of everything we do including in our external influencing work is embedded in the organisational strategy at Freedom from Torture, so we have been lucky enough to have ring-fenced resources for a number of years. 


\section{Activism groups and activists}

Survivor activists in Freedom from Torture projects are men, women and young people who have been tortured and are either asylum applicants or refugees in the UK. They are all current or former clients of Freedom from Torture. They are supported by a team of volunteers and staff and together we have grown survivor activism into an area of work that is now acknowledged within the organisation as an important part of the rehabilitative journey for survivors of torture. The three groups, two adult and a third being set up for young people, focus on promoting survivor-led or supported interventions in public spaces, policy debates and practice about torture, but they all have different ways of working.

\section{Survivors Speak OUT (SSO)}

" $A$ s an activist, I can address some of the injustices of my past and present. For too long others told my story but now I am speaking for myself and for other torture survivors. We are taking back control and working hard to make sure that we are part of finding and shaping solutions about our lives", Serge Eric Yamou, co-founder of SSO, and now Trustee at Freedom from Torture Survivors Speak OUT is a survivor-led activist network supported by the charity but which was in fact initiated by a small group of service users ${ }^{2}$ who had a passion to speak out against torture and its impact. The network is now ten years old. There are more than 30 active members all of whom have completed treatment at one of Freedom from Torture's five

2 A user of Freedom from Torture's rehabilitation services centres (London \& South East, Glasgow, Manchester, Newcastle and Birmingham). Recently, their work has focused on direct advocacy with domestic and international decision makers like the UK Home Office, the Foreign and Commonwealth Office and cross-party governmental scrutiny bodies, but its members also speak at events in the UK and abroad including at colleges and universities, in Parliament, and to the United Nations, to raise awareness and press for change.

The group speaks as a network of survivors with one voice. This strengthens their message but also supports their own personal well-being. A driving philosophy of their work is to let people know that as survivors of torture, they are not simply a source of testimony but that their lived experience of torture and its impact means they have crucial insights to shape change and create meaningful solutions.

Examples of their impact include staff training implemented in the UK Home Office's Asylum Intake Unit about greater awareness of behaviours and attitudes on asylum applicants. This training was implemented after joint advocacy work by SSO and Freedom from Torture with the Intake Unit; and the introduction of a principle in the UK Preventing Sexual Violence Initiative's (PSVI) Global Principles for Action to tackle Sexual Violence in Conflict on empowering survivors to speak out.

As an organisation, we support the network by helping members to access opportunities to influence others. The process of support is intensive and includes capacity building for their public speaking, media training and advocacy skills, preparation through workshops to support them to develop policy positions and key messages, help to write speeches 
and articles, work to develop and promote their brand as a network, and also as individual advocates. In addition, there is a need to develop and manage safeguarding, good practice around a range of issues including individual security and self-care. Throughout this process the focus is always on supporting network members to develop their identities as activists so that they are recognised within their own right.

SSO has taught us the power of speaking with a collective voice. This approach sometimes stops survivors being included in discussions and debates because those reaching out to the group are interested only in testimony about specific torture experiences. But this model has allowed us to help the Survivors Speak OUT network to develop collaborative policy positions which helps to reduce the risk of personal credibility attacks and protects the network from claims of bias in relation to a country or an issue. We have also taken partners along with us on this journey-for example, we convinced the UK Foreign \& Commonwealth Office that survivors could contribute more than their stories. The coordinator of the network now serves on the ministerial steering group for the UK's Preventing Sexual Violence in Conflict Initiative. This approach also enables survivor perspective on human rights issues to inform the influencing programmes and policy positions of Freedom from Torture.

\section{Write to Life}

"Writing to me is activism-it makes me feel who I am"... "I write to empty myself of my memories, to choose things to remember and challenge myself to move on", Quotations from Survivor Activists participating in Write to Life
Write to Life, ${ }^{3}$ our creative writing and more recently performance group, is now 20 years old. The group began as a therapeutic space based in our clinical department. It has now evolved into a group supported by professional writers who work with its members and support them to reach out to new and previously untapped audiences through poems, films, music and theatre, most of which the members perform themselves. Unlike Survivors Speak OUT, members of Write to Life are both current and former service users. Again, an important principle for the group is that they do not recount torture experiences but find creative and engaging ways to tell their versions of their stories, whatever that may be, on their terms.

One of Write to Life's key achievements has been through transforming stories and words into artistic forms of selfexpression that has enabled new audiences to be reached that might not otherwise be interested in torture and its' impact. An example of this is the play, Souvenirs, that was written and performed by members of the group (all survivors of torture) which toured theatres to packed-out audiences. One audience member said, "Souvenirs burned itself on my soul. Not in any way bleak or sympathy stealing. It felt like a true human connection between audience and performers. This is the most important thing I have seen a theatre do."

Appropriate safeguards to reduce risks and respond to incidents that might impact mental health must continuously be developed, improved and implemented. Another current focus is promoting collaborative approaches between group

\footnotetext{
3 https://www.freedomfromtorture.org/survivor
} activism/write_to_life 
members and support staff/volunteers. We strive to find a balance in providing enough space for the group to nurture its creative approach that enriches writing and performance. At the same time, we also look at how their work can support the strategic policy priorities set by the organisation in collaboration with survivors, for example through performances for advocacy targets or creative projects aligned to campaign objectives.

\section{Youth Voices}

The third group, Youth Voices, is for younger torture survivors aged 16 to 25 who have an interest in activism. The purpose of this group is to create a space for young survivors to have a more prominent voice and speak out on issues that impact their lives in the UK. Young people in the UK experience the asylum system and other services differently and too often their voices are not heard over adult interests. The direct engagement phase with young people begins in summer 2018 .

\section{What does it take?: Dedicated resources}

Enabling engagement: When people (survivor activists) join our groups, it begins with an induction about the group, its members, what is involved, a two-way discussion about expectations, how to make a complaint and a set of policies to support good and safe practice. It is important to be clear that survivor activism is not a clinical group or a formal part of the therapeutic process because it is of course essential for group members to know if they are a therapy client for a number of reasons; ethically they need to be clear about their relationship with the organisation; therapy clients may have access to different internal services; and staff in survivor activism are not clinical therapists although there are situations where we are supported by clinical staff.

Nonetheless, some people see our survivor activism work as an important part of their rehabilitation journey and writing like that of Write to Life certainly has therapeutic benefits. Elif from Write to Life describes how writing enables distance from a painful past and allows me to focus on the present and the future.

One important way to support engagement in activism is by holding regular group meetings for survivor activists to come together, discuss issues, reach common agreement and generally bond as a group with a common cause. This is important because our groups are made up of women and men from different backgrounds, cultures, expectations and experiences so taking the time to understand each other is important

It is important to inspire people to engage through skill-building workshops that are relevant to them and that they enjoy. These workshops might involve support to members of our groups to formulate policy positions, recommendations for change, write speeches or poems. If survivor activists are volunteering their time and energy around initiatives related to deeply personal and traumatic issues, they need to feel the value of their engagement.

People are supported to express themselves in their own words, avoiding jargon and using simple language. They don't need to speak the best English but they will have to have some level of English to speak to their audience directly - they may need support to do this even though it in itself can create challenges around authentic voice and feelings of frustrations when someone cannot clearly express themselves in the way that they want or need to.

All of the activities and events we help the groups to participate in are 
supported by briefings as a group before, if necessary during, and always after, because preparation, preparation and more preparation is essential for impact. This approach creates confidence and also fosters an environment for reflection and learning and in turn, progression. Things can and will go wrong so a key part of my role is to facilitate a process of checking in throughout and supporting the group to make a decision together about next steps.

Involvement with survivor activists means that we develop long-term relationships through activism, in a way which for obvious reasons is rarely experienced in the more boundaried clinical environment. That means that we see the lasting impact of torture, exile, rehabilitation and survival. This unique perspective supports our work with survivor activists because we often understand the issues and the drivers that they prioritise. It also means that we learn of, and are sometimes called on to provide support on legal and welfare issues they are experiencing. At Freedom from Torture we are fortunate to have specialist colleagues in house to give legal advice and/or signpost to welfare support services, when they have capacity. This is an issue we continue to explore internally given limited resources and the need to prioritise those with the most need while also wanting to meaningfully support the people we have long-term relationships with.

"Black writing ran wildly around the page like spiders. All I could see was the word 'refusal'. I lost my courage. I could not continue to read. The world had gone blue. My solicitor read out the rest”, Extracts from 'The Letter' by Anon from Write to Life
People: A dedicated team makes it possible to build relationships, develop trust and, as a result, is more effectively able to build knowhow, appropriate polices, and good practice to monitor the work and ensure that it is safe and as risk free as it can be for survivor activists to engage.

A key element of the success of survivor activism at Freedom from Torture is the existence of a dedicated team which supports the work, as well as a ringfenced set of organisational resources. This commitment is embedded in our organisational strategy to put survivors of torture at the heart of everything we do including in decision making.

There has been a conscious decision to ensure that this is not extra work tagged on to that of all or other teams but a body of work in itself. This is of course important because survivor activists are still potentially vulnerable people living with deeply traumatic pasts and in many instances very difficult present circumstances. They are highly motivated to engage in this work for a number of reasons including so that others do not face what they have had to, but part of the journey for them, and for us, can involve exploring issues which they may not have had cause to address in therapy or other support, for example the potential to meet a government representative from their home country in an international meeting.

Operational budgets: Activities for the groups can be costly. Realistic and dedicated budgets for the day-to-day running of the work are important. Money is needed to bring people together, to pay for skillsbuilding initiatives and to attend conferences (e.g. within the charity, homelessness and/ or health sector), and provide basic costs to enable practical involvement of activists, including travel fares and costs related to the 
provisions of childcare. This has significant cost implications but also challenges in ensuring the implementation and navigation of a transparent system. Despite this, a conscious decision has been made not to replicate the barriers that survivor activists face in their day-to-day life because of their gender and/or their asylum status. We believe this is also essential to move survivor engagement away from tokenistic models into meaningful engagement because it allows for sustained development of skills, and gives the groups the opportunity and time to develop thoughtful advocacy positions. In our view, this means that we have to break down the barriers that prevent meaningful engagement in a progressive way that leads to up-skilling and growing confidence (as opposed to passive involvement). We are fortunate in the UK that, as well as the support of the organisation, there are a number of funders that share our vision and support this work.

Good practice: The survivors we work with are without a doubt incredibly resilient, but it is also important to recognise that they are also susceptible to setbacks, not only because of the torture they have survived and its long term impact on their lives, but also due to the myriad of challenges faced living as an asylum seeker or refugee in the UK. Survivor activism by its nature more often involves exposing survivors and their experiences to strangers on platforms in the UK and internationally. Together these issues can bring a host of challenges and risks.

Measures need to be in place to ensure that our day-to-day work is based on safe practices and guides staff and activists to respond appropriately when faced with these challenges. It is not about wrapping people up in cotton wool but as an organisation taking our duty of care in a survivor activism context seriously.

It is important that informed consent is based on a thorough assessment to support survivor activists to navigate the risks and benefits associated with involvement in an activity. It should also include information on how to withdraw consent and there should be a responsibility on the staffer or facilitator to ensure that they have explained it clearly, discussed the appropriate risks and benefits, and follow through to make sure an individual's choices are respected.

Preparation for activities should always include gathering enough information to carry out a risk assessment without which the informed part of consent (permission based on full knowledge and consequences) can't be given by survivor activists.

Participants need to clearly understand what will happen to the information they share, where it might be used and what exposure it will be subject to including on social media and the internet. Exposure on digital channels opens up other issues to consider including issues around stigma, the potential for their experience to reach a far wider audience including in their home country with implications for family members wherever they might be, and more.

That is why for survivors of torture navigating consent is not about focusing simply on the present but the future too. The increasingly hostile efforts of states towards refugees seeking protection means that we do not know if they might face return to a torturing state either on refusal of asylum status or renewal of refugee status. Our responsibility to the well-being and safety of survivor activists is paramount and overrules our need to be relevant in debates or raise organisational profile.

Another essential tool is the Critical Incident Protocol which allows us to 
respond to situations that result in survivor activists experiencing a serious deterioration of mental health including thoughts of suicide, and any child protection issue. This might be the result of a particular activity or something external to the work. The protocol sets out clear guidance on how to spot these signs and respond to situations both in and outside of working hours for non-clinical staff in the survivor activism team. It is as much about supporting the person experiencing a mental health set-back as it is for the staffer or activist responding to the incident. Having such a protocol in place is essential not only for the well-being of individuals but also as a safeguard for the organisation which may have legal and other questions to face in such a situation from regulatory bodies as well as others.

There are also other policies and practices implemented to respond to other seemingly simple but in reality more complex issues. This includes communications within the activism groups on online platforms such as social media or mobile apps where information and video footage moves around at a rapid pace and can include graphic and distressing content; and approaches between and amongst staff and survivor activists to maintain clear and professional boundaries in the context of friendships born out of this work often outside of core working hours.

\section{Strategic development: Survivor- centred to survivor-led}

"I may have lost some dignity and respect

I was once treated with, but I want to say that in all that you do, always remember the words and contributions of women survivors of torture. Our pasts may have left us in shock and in pain but we are resilient. I now speak out against torture and its impact and that takes courage and it means that I control my own narrative and for me, that is important", Tracy N'dovi, Survivor Advocate, SSO

Working with and not just for survivors is an important principle, especially within an organisation with day-to-day contact with survivors of torture. Before being able to create an opportunity to work alongside survivors in a meaningful way, an environment that supports people to build their skills and capacity to engage in activism and feel confident about speaking out needs to be created.

We do this by putting survivors and the impact of their experiences at the centre of our approach. This involves working with people to understand how they process information, how their experiences of torture impact their engagement, what the environment needs to feel and look like to enable engagement, whether there are barriers that inhibit engagement, how factors like people, gender, status and culture influence involvement, and crucially what needs to be in place to encourage and motivate people to join discussions and debates.

Ultimately, the goal is to move to a survivor-led approach. This is a long-term aspiration in which investment is put into supporting survivors to lead on their own organising, their own facilitation, and their own activism whatever that may look like. However, it is important to realise that even when survivors have been politically active in other contexts, such as their home countries, this may take time. For example, it is important to explore and understand different advocacy spaces given that some of the survivor activists were human rights defenders in their home countries. Looking at cultural differences in influencing public audiences or decision makers in the UK or at international fora like the United Nations, 
and finding common ground is a critical part of capacity building.

The survivor-led approach is about supporting people to take more control and responsibility to deliver the impact that they have identified. In practice, this is resourceintensive and involves commitment from the survivor group together with the skills and know-how to carry out parts of the day-today work. There are also some more thorny issues of accountability to other group members, funders, the host organisation and others. The reality is that ultimately you are working with people and not all people conform to a particular approach that might be needed by the organisation. These are issues that arise day to day in every work place and this area of work is no exception.

From experience, we know that this approach might not always or consistently work; we are grappling with and exploring different approaches, and this has value in itself. For example, whether to include survivor activist in work behind the scenes like workshop design or facilitation, work closer to delivery stage including speech development and delivery, or both whilst considering capacity in terms of availability, time, skills and budget. As an organisation it is important to be prepared to be flexible about the type and level of support that is needed.

\section{Learning along the way}

Monitoring and evaluation: Good practice should include having mechanisms in place to evaluate this work both in terms of benefits to survivor activists and shaping more progressive ways to engage survivors in influencing opportunities, but also for policy impact. We do continuous evaluation through listening to survivor activists at the meetings and workshops mentioned above but we do not do circulate evaluation forms after each event; when a group is strong, individual feedback forms are often not wanted. Instead, creating a climate of trust and building confidence means that survivor activists will express themselves when they need to. And this works two ways in a mutually respectful relationship. All of this information is collated and fed back into the development of the projects. Unfortunately, we have not yet had the resources to carry out any clinical evaluations as a result of our projects. Certainly, for many of the survivor activists, although anecdotal evidence, they report that their involvement has led to an increase in their skills and confidence, and many have since secured employment including in engineering, academia, as interpreters, writers and authors, and as well as organisations like Freedom from Torture. Additionally, Freedom from Torture carried out a survivor-led six month study in 2016 involving over 100 clients to explore the meaning of rehabilitation to survivors. Some clients reported that feeling empowered to speak out about what had happened to them, and advocating for other survivors with decision makers or to public audiences, was a key part of rehabilitation. "They said that using their voice empowers them and helps restore their confidence. This is especially important for people who previously felt unable to use their voice to speak out in public." (Haoussou, 2018 p. 69)

The number of survivor activists who have engaged with us over the years is small in comparison to the number of clients at Freedom for Torture. This reflects that survivor activism is still a comparatively new area of work, and the resources we currently have reflect the capacity we have to deliver our work safely.

Recommendations: The wealth of learning is not possible to share in its entirety 
here but brief issues to highlight for other organisations looking to build similar models are:

- Few, if any, of the survivors now engaged in activism came as professional public speakers, advocates or performers. Yet because of torture, they are now doing just that so they need significant support. Take the time to invest, support and build trust;

- Support people to control their own narratives. Help them to or directly resist requests purely for testimony and focus on the messaging and positive action that are important to survivor activists;

- Try to secure dedicated resources to support survivor activism rather than adding this approach to other posts;

- Make sure you are able to spot risk including signs of deteriorating mental health and have the appropriate safeguards in place to respond appropriately;

- As in all groups that work together and can form strong bonds, there will not always be agreement; sometimes there will be falling out. Implement fair and appropriate support and responses to help navigate out of the situation;

- Don't let engagement be tokenistic, make it meaningful and when you're not able to do that, think about if it is fair to continue;

- Don't be afraid to give constructive feedback when things don't go to plan as learning is important. But consider the individual first and tailor your approach so that they don't feel demotivated. And of course, always praise when it all goes well;

- Keep an eye on individual and organisational egos (we all have them) so that the work remains balanced, fair and everyone's voices are amplified;

- Monitoring and evaluation in collaboration with survivor activists is important for progress, learning and funders; and

- We all need to find ways to share good practice and embed survivor activism in the anti-torture movement, in keeping with the human rights principles of empowerment and participation.

How to do this work with fewer resources: It is still possible to support survivor engagement in activism with fewer resources, but be realistic about what you can and can't do. The list below are some initial points to think about when considering this work for the first time:

- Have a clear sense of resources including staff time, skills in-house and budget;

- Have a clear and simple strategy with an aim (what do you want to achieve) and objectives (what must happen to help you achieve your aim);

- You must be able to deliver your strategy within existing resources and crucially, with buy-in and support from survivor activists and other support staff;

- Develop guidance to support good practice and at a minimum, be clear about gathering 'Informed Consent' that includes a thorough risk and safety assessment; and have a plan in place about how you will respond in the event of an emergency or risk to safety/ well-being;

- Be clear about what the engagement can and can't offer-be realistic;

- Start preparing for the activity and put in practice time before hand;

- Make sure that appropriate support is in place during the activity and debrief afterwards (to survivor activists and staff/volunteers); 
- Monitor progress and record well-being and any other risk issues that emerge;

- Consider your plan for next steps including assessing impact; and

- Maintain good boundaries throughout the process.

\section{Conclusions}

For organisations interested in building similar survivor activism models, this is a small offering of advice based on my personal perspective of what is necessary to consider to move any involvement away from tokenistic participation to a survivor-centred space.

There is a lot of hidden work that goes on behind the scenes including constant reflection about how to build on and improve for the future. Of course there are times where we might get it wrong. There are also times where internally our approach might not be in sync with other departments. But this is constant work in progress and our organisation continues to demonstrate its commitment to amplifying survivor voices including through survivor activism where we continue to build on our successes and look to grow over the coming years.

\section{References}

Haoussou K., (2017) Perspective-The long journey to rehabilitation for torture survivors. Torture 27 , (1) 66-74. 